\section{Movement aftereffects contingent on color, intensity, and pattern*}

\author{
J. E. W. MAYHEW and S. M. ANSTIS \\ University of Bristol, Bristol BS8 $1 \mathrm{HH}$, England
}

We have found contingent movement aftereffects (CMAEs) lasting several days, contingent upon the color, intensity, and stripe width of moving patterns. Ss adapted for $10 \mathrm{~min}$ to a patterned disk rotating clockwise under red light, alternating every $10 \mathrm{sec}$ with counterclockwise under green light. When stopped, the disk then appeared to rotate counterclockwise under red light and clockwise under green light. The effect lasted only a second or two, reappearing each time the field's color was changed. But it increased in strength over the first $1 / 2 h$ and was still present 1 or 2 days later. Color transposition effects were found: after adaptation to red-clockwise (long wavelength) alternating with green-counterclockwise (short wavelength), a stationary yellow (long wavelength) test field appeared to rotate counterclockwise and a blue (short wavelength) field appeared to rotate clockwise. Relative, not absolute, color of the test triggered the CMAE. Similar CMAEs and transposition effects were produced by pairing direction of movement with intensity, with width of moving stripes and with orientation of a stationary grating projected onto a rotating patterned disk.

It is well known that prolonged inspection of such visual stimuli as color, movement, dynamic change of brightness, and gratings of particular orientations or spatial frequencies causes visual adaptation during inspection and negative aftereffects on subsequent viewing of a neutral test field (Gibson, 1933, 1937; Holland, 1965; Anstis, 1967; Blakemore \& Sutton, 1969). These phenomena are simple aftereffects.

McCullough (1965) discovered the first of a new class of effects, which are called contingent aftereffects (CAEs).

She produced two aftereffects simultaneously by adapting Ss to vertical stripes against an orange background alternating with horizontal stripes against a blue background. In the test condition, Ss reported a blue-green aftereffect when viewing a neutral vertically striped pattern and an orange aftereffect on a neutral horizontally striped pattern. The color of the aftereffect was dependent on the orientation of the stripes on the retina, since tilting the head 90 deg down to one side caused the colors to switch from one pattern to the other. McCullough attributed the effects to the selective chromatic

\footnotetext{
*The authors thank Pamela Vaughan for her assistance in conducting the experiments, Brian Rogers for his helpful comments and discussion, and Tania Morley for drawing Fig. 1. They gratefully acknowledge support from the Science Research Council under Grant B/SR/4836. Also, the authors recently learned that 0 Favreau, V. Emerson, and M. C. Corballis Favreau, V. Emerson, and $M$. C. Corballis similar to their own, to be published shortly in Science, They are grateful to these authors for making them aware of their results before publication.
}

adaptation of two populations of edge population of edge detectors for vertical lines had been adapted to orange, while the population of detectors for horizontal lines had been adapted to blue. Harris and Gibson (1968) confirmed that the McCullough effect was not an artifact produced by ordinary colored afterimages, but proposed that it was caused by selective adaptation of "dipoles," not of edge detectors. A "dipole" is defined as a unit that receives inputs from two different locations on the retina. Lines of different orientation will tend to stimulate different populations of "dipoles." Fidell (1970) measured the strength of the aftereffect produced by adapting to gratings whose orientations differed by varying angles. She found that the effect decreased with decreasing angular separations until it was minimal at $11 \mathrm{deg}$ of separation. This angular specificity of the effect matches that of cortical cells sensitive to orientation in the cat and monkey, so Fidell considered it to support an edge detector interpretation. Held and Shattuck (1971) reported a complement or converse to the McCullough effect: they induced two simultaneous tilt aftereffects in different directions, paired with and elicited by complementary hues. Harris (1970) found colored aftereffects, similar to McCullough's but specific not to orientation but to spatial frequency of stripes. The aftereffects depended on the retinal spatial frequency, not on the perceived spatial frequency as corrected by size Stromeyer and Mansfield (1970) found a colored aftereffect showing a detectors (Hubel \& Wiesel, 1962). The constancy. Hepler (1968) and relationship between the direction of movement and color. Colored aftereffects that lasted as long as 6 weeks were produced with patterns of black and white stripes that moved vertically, or with rotating spirals. During adaptation, each pattern alternated in direction periodically, one direction being paired with a red illuminant and the other with a green illuminant. When the moving patterns were later viewed in white light, Ss saw red or green aftereffects, depending on the direction of movement of the test field. A direction that, during adaptation, had been paired with a red illuminant now elicited a green aftereffect, and vice versa.

In this study, we have investigated contingent movement aftereffects (CMAEs) which were contingent on the intensity, color, or spatial frequency of the patterns used in adaptation.

The aftereffects reviewed above are classified in Table 2, together with those which are reported in this study.

As an aid to understanding, the conditions and results of our Experiments 1, 2, 5, 6, and 7 are shown diagrammatically in Fig. 1.

\section{SUBJECTS}

A total of 60 unpaid undergraduate and graduate students took part in our experiments. About one-third of these were experimentally naive and had not previously experienced any simple or contingent AEs. We noted no systematic differences between the responses of naive and experienced $\mathrm{Ss}$. Between 4 and 12 Ss took part in each experiment.

\section{MAES CONTINGENT ON COLOR EXPERIMENT 1}

There were four Ss in two groups of two each.

Apparatus

A disk of $18 \mathrm{~cm}$ diam (10 deg of visual angle) was viewed from a distance of $105 \mathrm{~cm}$ through a lightproof black tunnel which excluded all ambient light. The disk was of translucent white material with a black four-throw spiral superimposed upon an irregular network of fine black lines (Letratone, 102). During adaptation, the disk rotated at a speed of $5 \mathrm{rpm}$, alternating in direction every $10 \mathrm{sec}$. The speed of rotation was not sufficient to give rise to a noticeable

Table 1

\begin{tabular}{|c|c|c|}
\hline & $\begin{array}{l}\text { Black Part } \\
\text { of Pattern }\end{array}$ & $\begin{array}{l}\text { White Part } \\
\text { of Pattern }\end{array}$ \\
\hline $\begin{array}{l}\text { Bright } \\
\text { Medium } \\
\text { Dim }\end{array}$ & $\begin{array}{l}1.4 \log \mathrm{fL} \\
0.0 \log \mathrm{fL} \\
2.4 \log \mathrm{fL}\end{array}$ & $\begin{array}{l}2.4 \log \mathrm{fL} \\
1.0 \log \mathrm{fL} \\
1.4 \log \mathrm{fL}\end{array}$ \\
\hline
\end{tabular}




\begin{tabular}{|c|c|c|c|c|c|}
\hline \multirow{2}{*}{$\begin{array}{l}\text { Dimension of } \\
\text { Contingent } \\
\text { Aftereffect }\end{array}$} & \multicolumn{5}{|c|}{ Dimension of Eliciting Stimulus } \\
\hline & Color & Intensity & Movement & $\begin{array}{c}\text { Contour } \\
\text { Orientation }\end{array}$ & $\begin{array}{c}\text { Spatial } \\
\text { Frequency }\end{array}$ \\
\hline Color & (Afterimages) & & $\begin{array}{l}\text { Hepler, 1968: } \\
\text { Stromeyex \& } \\
\text { Mansfield, } 1970\end{array}$ & McCullough, 1965 & Harris, 1970 \\
\hline Intensity & & (Afterimages) & Yes & Yes & \\
\hline Movement & $\begin{array}{l}\text { Mayhew \& Anstis } \\
\text { (Experiments 1-4) } \\
\text { Favreau, Emerson, } \\
\text { \& Corballis, 1972 }\end{array}$ & $\begin{array}{l}\text { Mayhew \& Anstis } \\
\text { (Experiments 5-6) }\end{array}$ & $\begin{array}{l}\text { (Simple MAEs) } \\
\text { Reviewed by } \\
\text { Holland, } 1965\end{array}$ & $\begin{array}{l}\text { Mayhew \& Anstis } \\
\text { (Experiment 9) }\end{array}$ & $\begin{array}{l}\text { Mayhew \& Anstis } \\
\text { (Experiment } 7 \text { ) }\end{array}$ \\
\hline $\begin{array}{l}\text { Contour } \\
\text { Orientation }\end{array}$ & $\begin{array}{l}\text { Held \& Shattuck. } \\
1971\end{array}$ & & & $\begin{array}{l}\text { Gibson, 1933, } 1937 \\
\text { Campbell \& } \\
\text { Maffei, } 1971\end{array}$ & \\
\hline $\begin{array}{c}\text { Spatial } \\
\text { Frequency }\end{array}$ & & & & & $\begin{array}{l}\text { Blakemore \&x } \\
\text { Sutton, } 1969\end{array}$ \\
\hline
\end{tabular}

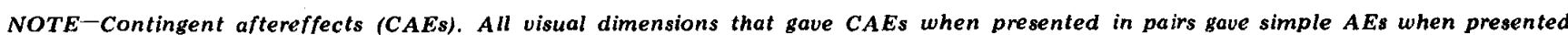

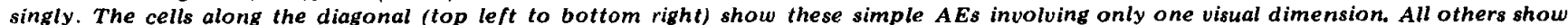

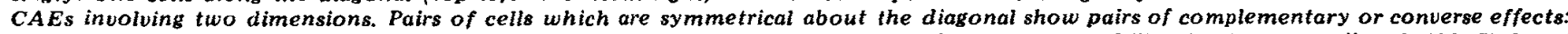

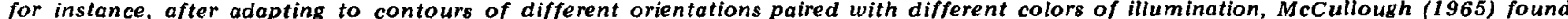

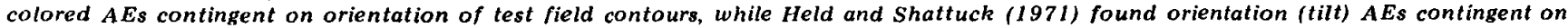
test field color. The origin of CAEs is not known.

expansion or contraction; however, the combination of the two patterns had been found to give an extremely large simple rotary MAE, and was used for this reason. The disk was back illuminated by projectors carrying colored filters as required.
Adaptation Procedure

A uniformly colored field was projected onto the rotating disk. The color of the field was changed each time the disk alternated in direction. Group 1 adapted to red-clockwise alternating with yellow-counter- clockwise. Group 2 adapted to yellow-clockwise alternating with green counterclockwise. The luminances and wavelengths were as follows: Red was produced with Wratten Filter No. 25, and luminance was $1.58 \log$ FL. Green was Wratten

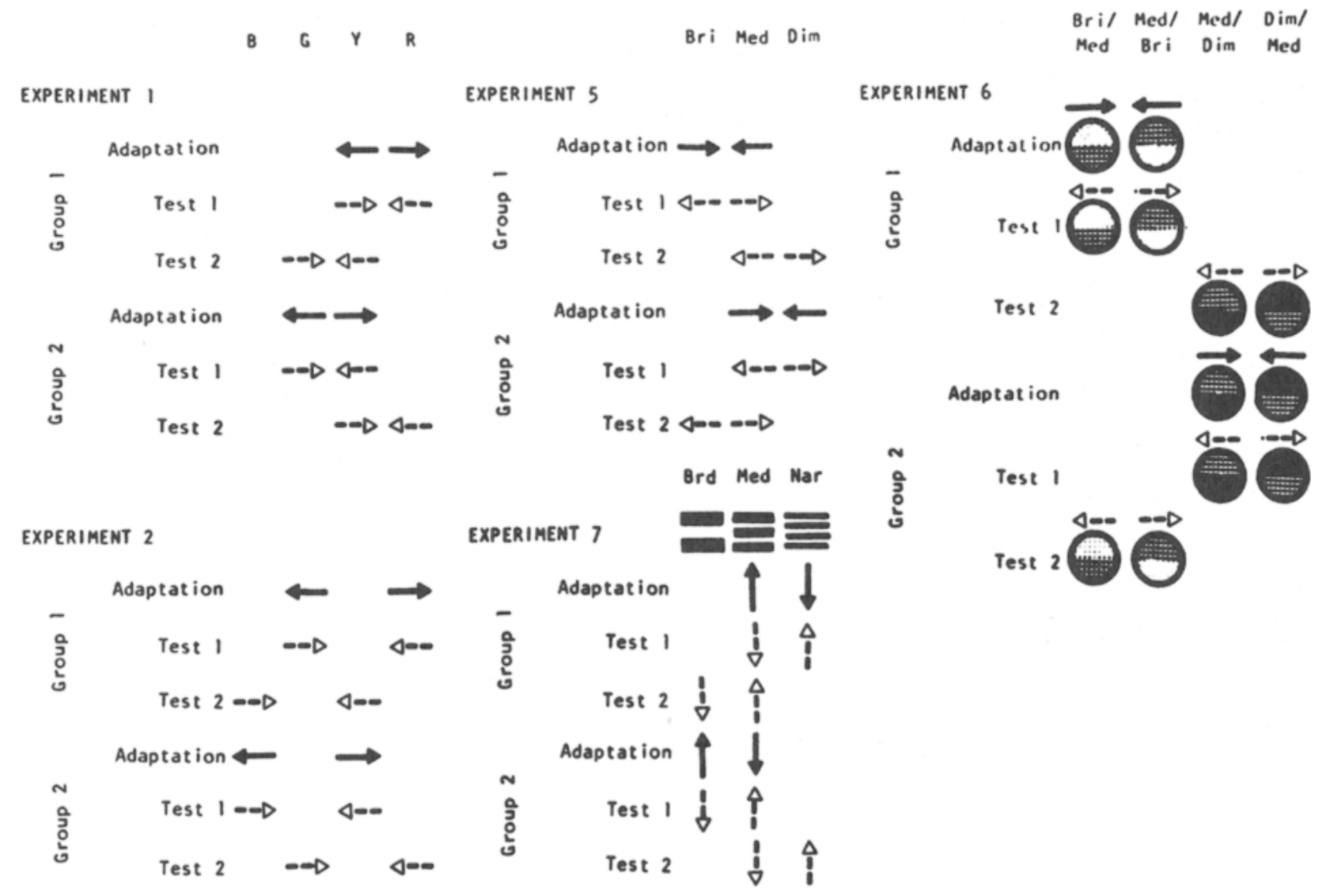

Fig. 1. Contingent MAEs: summary of conditions and results of Experiments 1, 2, 5, 6, and 7. In each experiment, Test 1 showed the paradigm CMAE and Test 2 the transposed CMAE. Experiment 6 included a Test 3 , not shown in the figure, for AEs of apparent brightness produced by a moving test field. $\rightarrow=$ clockwise rotation of adapting field; $\leftarrow=$ counterclockwise. $\cdots-D=$ clockwise $C M A E ; a \cdots=$ counterclockwise CMAE. $B=b l u e ; G=g r e e n ; Y=y e l l o w ; R=$ red; Bri = bright; Med = medium; Dim = dim; Brd = broad Med = medium; Nar = narrow . 
58, luminance $1.70 \log \mathrm{fL}$. Yellow was Wratten 12 plus 1 log unit ND, luminance $1.61 \log \mathrm{fL}$.

After $10 \mathrm{~min}$ adaptation there was a test procedure (Test 1) followed by $1 \mathrm{~min}$ rest, followed by a further 10-min adaptation and another test procedure (Test 2). During the last $30 \mathrm{sec}$ of the adaptation procedure, the rate of alternation of the stimulus fields was increased to one every 5 sec. This control prevented the buildup of a large simple MAE on the last exposure and was used in all our adaptation procedures.

\section{Test Procedure}

Two test procedures were used with stationary test fields. Before these were presented, Ss were informed that the movement of the fields would be stopped and the colors of the stationary fields would be alternated every 5 sec. They were required to comment on the apparent movement (if any) of the stationary test fields, using the responses "clockwise," "counterclockwire," or "stationary."

Test 1 was for the paradigm CMAE. It was given on the stationary adapting fields. Test 2 was for the transposition of the CMAE. ${ }^{\text {Thus, for } a} S$ in Group 1, Test $i$ exposed a stationary red field alternating with a stationary yellow field, and Test 2 exposed a stationary yellow field alternating with a stationary green field. For $a$ S in Group 2, the same tests were given but with the colors, of course, in the reverse order, i.e., Test 1 exposed yellow and green fields and Test 2 exposed red and yellow fields. As can be seen, each adapted to the stimulus fields that would be used as the transposition test fields (Test 2) for the other groups, so Test 1 and Test 2 were logically the same for both groups. This procedure ensured that the transposition test fields were, at least in principle, capable of keying or eliciting CMAEs. In both tests, the fields were alternated approximately every 5 sec. So were then given Test 1 and their responses recorded. Ss were judged to have experienced a paradigm CMAE if they consistently reported MAEs in the appropriate direction on 10 successive alternations of the test fields. Ss were then given a 1-min rest and a further period of adaptation, after which Test 2 was administered. Transposition was judged to have occurred if during Test 2 Ss consistently reported a MAE on the yellow field opposite in direction to that reported during Test 1 . The adaptation and test stimulus conditions can be summarized as follows:

\section{Group}

Adaptation. Red-clockwise alternating with yellow-counterclockwise every $10 \mathrm{sec}$.
Test 1 . Red-stationary alternating with yellow-stationary every 5 sec.

Test 2. Yellow-stationary alternating with green-stationary every 5 sec.

\section{Group 2}

Adaptation. Yellow-clockwise alternating with green-counterclockwise every 10 sec.

Test 1 . Yellow-stationary alternating with green-stationary every $5 \mathrm{sec}$.

Test 2. Red-stationary alternating with yellow-stationary every 5 sec.

\section{Results}

Test 1. All Ss reported paradigm contingent MAEs. Group 1 Ss reported counterclockwise MAEs on the red test field and clockwise MAEs on the yellow test field, and Group 2 Ss reported counterclockwise MAEs on the yellow field and clockwise MAEs on the green field.

Test 2. All Ss reported transposed CMAEs. Group 1 Ss now reported a counterclockwise MAE on the yellow test field and a clockwise MAE on the green field, and Group 2 Ss reported counterclockwise MAEs on the red field and clockwise MAEs on the yellow field.

The MAEs were contingent purely on the temporal order in which the colors were presented. When a yellow field was presented to either group, it appeared to move clockwise when it came after red (paradigm CMAE for Group 1, transposed CMAE for Group 2) and counterclockwise when it came after green (transposed CMAE for Group 1, paradigm CMAE for Group 2). The direction of the CMAE depended not on the absolute color of the test field, but on the relative change in its color. It was clockwise when the test field changed to a shorter wavelength (red changing to yellow) and counterclockwise when it changed to a longer wavelength (green changing to yellow). This was demonstrated very markedly when the following test was given after completion of Tests 1 and 2 . Both groups of Ss were presented with the following sequence of test fields: red-yellow-green-yellow-red-yellowgreen-yellow, etc., and Ss typically responded "counter, clock, clock, counter, counter, clock, clock, counter," etc., where it can be seen that after a red field yellow keyed a clockwise MAE and after a green field yellow keyed a counterclockwise MAE.

After completion of the test procedures, the Ss were asked to describe the effects. CMAEs were reported as a small transient apparent motion of the field, lasting from 1 to $2 \mathrm{sec}$ after the fields had been changed. They were, however, very durable in that these brief effects could be elicited time and time again over a period of hours or days.

\section{EXPERIMENT 2}

There were four Ss in two groups of two each.

This experiment controlled against a possible artifact in the color transposition effect. The authors thought it possible that during adaptation in Experiment 1, color afterimages might build up and corrupt succeeding adapting fields. For example, for Group 1 Ss the red adapting field might build up a green afterimage that would tinge the succeeding yellow adaptation field. Similarly, for Group 2 Ss, the green adaptation field could build up a red afterimage that tinged the succeeding yellow adaptation field. A similar corruption of successive fields could have occurred in the test conditions. Experiment 2 investigated the transposition of color-contingent CMAEs using colors specially chosen to ensure that this artifactual broadening of the adaptation spectrum did not underlie the effects.

\section{Apparatus}

The apparatus used was as described in Experiment 1.

\section{Adaptation Procedure}

A uniformly colored field was projected onto the rotating disk. The color of the field was changed each time the disk alternated in direction. Group 1 adapted to red-clockwise alternating with green-counterclockwise. Group 2 adapted to yellow-clockwise alternating with blue-counterclockwise. The wavelengths and luminances were as follows: Red was produced with Wratten Filter No. 25 and had a luminance of $0.65 \mathrm{log} \mathrm{fL}$. Yellow was Wratten Filter No. 12, luminance $0.65 \mathrm{log} \mathrm{fL}$. Green was Wratten Filter No. 58, luminance $0.62 \log$ FL. Blue was Wratten Filter No. $47 \mathrm{~b}$, luminance $0.52 \log \mathrm{fL}$.

After $10 \mathrm{~min}$ adaptation, there was a test procedure (Test 1) followed by a 1-min rest, followed by a further 10-min adaptation and another test procedure (Test 2 ).

\section{Test Procedure}

As in Experiment 1, two test procedures were given: Test 1 for the paradigm CMAE and Test 2 for the transposition of CMAEs. For a $S$ in Group 1, Test 1 exposed a stationary red field alternating with a stationary green field and Test 2 exposed a stationary yellow field alternating with a stationary blue field. For a $S$ in Group 2, Test 1 exposed a stationary yellow field alternating with a stationary blue field and Test 2 exposed a stationary red field 
alternating with a stationary green field. The other details of the testing procedure were the same as described in Experiment 1. The adaptation and test stimulus conditions can be summarized as follows:

$$
\text { Group I }
$$

Adaptation. Red-clockwise alternating with green-counterclockwise every 10 sec.

Test 1. Red-stationary alternating with green-stationary every 5 sec.

Test 2. Yellow-stationary alternating with blue-stationary every 5 sec.

$$
\text { Group } 2
$$

Adaptation. Yellow-clockwise alternating with blue-counterclockwise every 10 sec.

Test 1. Yellow-stationary alternating with blue-stationary every 5 sec.

Test 2. Red-stationary alternating with green-stationary every 5 sec.

\section{Results}

The results are shown diagrammatically in Fig. 1.

Test 1. All Ss reported paradigm CMAEs. Group 1 Ss reported counterclockwise MAEs on the red test field and clockwise MAEs on the green test field. Group 2 Ss reported counterclockwise MAEs on the yellow test field and clockwise MAEs on the blue test field.

Test 2. All Ss reported transposed CMAEs. Group 1 Ss reported counterclockwise MAEs on the yellow test field and clockwise MAEs on the blue test field. Group 2 Ss reported counterclockwise MAEs on the red test field and clockwise MAEs on the green test field. After completion of Test 2 , Ss in both groups were retested on Test 1 . They were asked to comment on the relative strengths of the paradigm and transposed CMAEs. They reported the transposed CMAE to be weaker than the paradigm CMAE.

In this experiment, the colors of each pair of adapting and test fields were complementary. The colored afterimages built up during exposure to either a test or adapting field served only to intensify and not to corrupt successive fields. From the results of this experiment, we conclude that the transposition of color-contingent MAEs is not the result of artifactual broadening of the adaptation spectrum.

\section{EXPERIMENT 3}

There were six Ss in two groups of three each.

This experiment controlled against the possibility of a brightness artifact. Since the colors we used were not exactly matched for brightness, it was possible that this brightness difference, not the color difference, was eliciting the CMAEs.

\section{Apparatus}

A disk of $30 \cdot \mathrm{cm}$ diam (6 deg of visual angle) was viewed from a distance of $2.9 \mathrm{~m}$. The disk was covered with $0.5-\mathrm{cm}$-diam black dots arranged irregularly. The overall black-white ratio was 1:10. The disk was front-illuminated by projectors carrying colored filters as required and was centered in a stationary white screen (luminance $1.15 \log \mathrm{fL}$ ), $45 \mathrm{~cm}$ ( 9 deg of visual angle) high and $70 \mathrm{~cm}$ (14 deg of visual angle) wide. The disk rotated at $8 \mathrm{rpm}$, alternating in direction every $10 \mathrm{sec}$.

\section{Adaptation Procedure}

A uniformly colored field was projected onto the rotating disk. The color and luminance of the field were changed each time the disk alternated in direction. Group 1 adapted to dim-red-clockwise alternating with bright-green-counterclockwise. G r o u p 2 a d a p t e d to bright-red-clockwise alternating with dim-green-counterclockwise. The wavelengths and luminances were as follows: Red was produced with Wratten Filter No. 25; bright-red had luminance of $1.03 \log \mathrm{fL}$, and dim-red had a luminance of $0.47 \log \mathrm{fL}$. Green was produced with Wratten No. 58; bright-green had a luminance of $1.16 \mathrm{log} \mathrm{fL}$, and dim-green had a luminance of $0.62 \log \mathrm{fL}$. After $10 \mathrm{~min}$ adaptation, there was a test procedure, followed by a 1-min rest and a further test procedure.

\section{Test Procedures}

There were two test procedures for each group. Test 1 was for the paradigm CMAE and was given on the stationary adapting fields. Test 2 was given as a brightness control. The colors of the fields were the same as in Test 1 , but their relative brightnesses were reversed.

The adaptation and test stimulus conditions were as follows:

$$
\text { Group } 1
$$

Adaptation. Dim-red-clockwise alternating with bright-green-counterclockwise every 10 sec.

Test 1 . Stationary-dim-red alternating with stationary-brightgreen every 5 sec.

Test 2. Stationary-bright-red alternating with stationary-dim-green every 5 sec.

\section{Group 2}

Adaptation. Bright-red-clockwise alternating with dim-green-counterclockwise every 10 sec.

Test 1. Stationary-bright-red alternating with stationary-dim-green every 5 sec.

Test 2. Stationary-dim-red alternating with stationary-brightgreen every 5 sec.

As in Experiment 1, the Ss were judged to have experienced a CMAE if their reports showed no inconsistency and a minimum of 10 successive MAEs in the appropriate directions were reported.

For all Ss, red elicited a counterclockwise CMAE and green elicited a clockwise CMAE, irrespective of the brightness differences of the fields. The brightness differences in this experiment were of the order of 0.5 $\log$ units, and a reference brightness was provided in the form of an illuminated surround to the adapting and test fields. In Experiments 1 and 2 , all differences in brightness of the stimulus fields were less than 0.2 log units and no reference brightness was present. We conclude that the CMAEs reported in Experiments 1 and 2 were truly contingent on color and not on brightness.

All the CMAEs reported in this paper were much briefer and less vivid than ordinary simple MAEs. They appeared only briefly for 1 to $2 \mathrm{sec}$ after each change of the test field. However, they could still be elicited several hours or even days after adaptation. We also found, as did Stromeyer and Mansfield (1970), that after a rest period of about $1 / 2 h$ between test and retest $S$ s reported a large increase in the size of CMAEs.

\section{EXPERIMENT 4}

This was carried out to show that the CMAEs were also remarkably resistant to extinction. Four Ss adapted for $10 \mathrm{~min}$ to red-clockwise alternating with green-counterclockwise. The apparatus and adaptation procedure were the same as in Experiment 1. All Ss reported paradigm CMAEs on stationary red and green test fields. They were "deadapted to color" by inspecting the stationary red and green test fields, which were presented alternately every 3 sec for a total of $15 \mathrm{~min}$ in three blocks of 5 min each. Between each of these "color deadapting" blocks, Ss were also "deadapted to movement" by $2.5 \mathrm{~min}$ inspection of the black and white pattern rotating clockwise under white illumination (white-clockwise) a Iternating every 3 sec with white-counterclockwise. Towards the end of this "deadapting" procedure, Ss reported a decrease in the size of the CMAEs when they viewed the stationary red and green test fields, but it was restored by a 2-min rest in the dark and was reported by two of the $S s$ as still present, though very small, 2 days later. CMAEs seemed to 
be very durable and indestructible.

\section{MAEs CONTINGENT ON BRIGHTNESS}

Just as adaptation to colored movement gave colored aftereffects on a neutral moving test field and movement aftereffects on a stationary colored field, so it was found that adaptation to moving fields of different intensities gave intensity aftereffects on moving test fields and movement aftereffects on stationary test fields of suitable intensities.

Pilot work showed that adapting fields, moving in opposite directions, needed to differ in intensity by $1 \mathrm{log}$ unit to produce CMAEs, but that test fields needed to differ only by $1 / 2 \log$ unit. To give large CMAEs, both our adapting and our test fields were made to differ by $11 / 2 \log$ units.

\section{EXPERIMENT 5}

There were six Ss in two groups of three each.

\section{Apparatus}

The apparatus was the same as in Experiment 1 for MAEs contingent on color. Briefly, the $S$ viewed a patterned disk $18 \mathrm{~cm}$ (10 deg of visual angle) in diam from a distance of $105 \mathrm{~cm}$ through a lightproof black tunnel. During adaptation, the disk rotated at a speed of $5 \mathrm{rpm}$, alternating in direction every 10 sec. It was back-illuminated by projectors carrying neutral density filters as required.

\section{Adaptation Procedure}

A uniform field of light was projected onto the rotating patterned disk. The brightness of the field was changed each time the disk alternated in direction. Group 1 adapted to bright-clockwise alternating with medi um-counterclockwise. Group 2 adapted to medium-clockwise alternating with dim-counterclockwise. The luminances of the illuminated spiral on the disk are shown in Table 1. After $10 \mathrm{~min}$ adaptation there was a test procedure followed by a 1-min rest, followed by a further 10-min adaptation and a second test procedure.

\section{Test Procedure}

The same test procedure was used as in the previous experiments. Test 1 was for the paradigm CMAE, and Test 2 was for the transposition of CMAE. The adaptation and test stimulus conditions were as follows:

$$
\text { Group } 1
$$

Adaptation. Bright-clockwise alternating with medium-counterclockwise every 10 sec.

Test 1. Bright-stationary alternating with medium-stationary every 5 sec.
Test 2. Medium-stationary alternating with dim-stationary every $5 \mathrm{sec}$.

\section{Group 2}

Adaptation. Medium-clockwise alternating with dim-counterclockwise every 10 sec.

Test 1. Medium-stationary alternating with dim-stationary every 5 sec.

Test 2. Bright-stationary alternating with medium-stationary every 5 sec.

\section{Results}

The results are shown diagrammatically in Fig. 1.

Test 1 . For all the $\mathrm{Ss}$, the brighter of the two fields consistently elicited a large counterclockwise MAE. However, Ss in Group 1 reported no MAEs on the dimmer (medium) field, and although two $S s$ in Group 2 did report a MAE on the dimmer (dim) field, it was judged very small and had disappeared before the fifth test exposure.

Test 2. Only one $\mathrm{S}$ from Group 1 reported a transposed MAE. Paradoxically, in the light of the results of Test 1 , he consistently reported a small clockwise MAE on the dim field but reported no MAE on the medium field. The other two Ss in Group 1 reported no MAE on either the medium or the dim field, yet when retested on Test 1 they consistently reported a MAE (counterclockwise) on the bright field. All the Ss in Group 2 reported transposed MAEs, but only when the test field made a sudden increase in brightness. When the test fields were alternated in the sequence of bright, medium, dim, medium, bright, medium, dim, etc., Ss typically responded "counterclock, nothing, nothing, counterclock, counterclock, nothing, nothing, etc." A bright test field gave a counterclockwise MAE, a dim test field gave none, and a medium test field gave a counterclockwise MAE when it followed a dim field but none when it followed a bright one. We have no explanation for this asymmetry.

\section{EXPERIMENT 6}

There were eight $\mathrm{Ss}$ in two groups of four each.

Apparatus
The apparatus was as described
above.

\section{Adaptation Procedure}

A field split in half along the horizontal diameter was projected onto the disk. The luminances of the two halves of the fields differed by approximately 1.5 log units. Each time the disk alternated in direction, the positions of the two halves of the field were reversed. Group 1 adapted to bright-above-medium-clockwise alternating with medium-above-brightcounterclockwise. Group 2 adapted to medi u m-above-di m-clockwise alternating with dim-above-mediumcounterclockwise. The figure and ground luminances were the same as in Experiment 1 . After $10 \mathrm{~min}$ adaptation, Test 1 was given followed by a 1-min rest and a further period of adaptation. This was followed by Test 2 and a further 5 min adaptation, after which Test 3 was given.

\section{Test Procedure}

There were three test procedures. Test 1 was for the paradigm CMAE and was given on the stationary adapting fields. Test 2 was a test for transposition, and the test fields were the stationary adapting fields of the other $S$ group. Test 3 was for an inverse effect, namely, the brightness analogue of the Hepler-Stromeyer effect. A homogeneous field divided by a thin stripe along the horizontal diameter was projected onto the disk, which alternately rotated clockwise and counterclockw ise, changing direction every $10 \mathrm{sec}$. Ss were required to comment on the relative brightness of the two halves of the field. For Group 1, the rotating test field was "bright" attenuated by 0.5 $\log$ units, and for Group 2 the rotating test field was "medium" attenuated by 0.5 log units. The adaptation and test stimulus conditions were as follows:

\section{Group 1}

Adaptation. Bright-above-mediumclockwise alternating with medium-above-bright-counterclockwise every 10 sec.

Test 1. Bright-above-medium-stationary alternating with medium-above-bright-stationary every 5 sec.

Test 2. Medium-above-dim-stationa r a lternating with dim-above-medium-stationary every 5 sec.

Test 3. Bright, -0.5 log units, alternately rotating clockwise and counterclockwise.

$$
\text { Group } 2
$$

Adaptation. Medium-above-dimclockwise alternating with dim-above-medium-counterclockwise every 10 sec.

Test 1. Medium-above-dim-stationa ry a l ternating wit h dim-above-medium-stationary.

Test 2 . Bright-above-medium-stationary alternating with medium-above-bright-stationary.

Test 3. Medium, $-0.5 \mathrm{log}$ units, alternately rotating clockwise and counterclockwise.

\section{Results}

The results are shown diagrammatically in Fig. 1. 
Test 1. Seven of the eight Ss reported paradigm CMAEs contingent on brightness. One $S$ in Group 1 reported no MAEs on either of the test fields, even after the second period of adaptation. He took no further part in the experiment.

Test 2. Six of the seven remaining Ss reported transposition of the CMAEs. One $S$ in Group 2 reported no transposed CMAE; this could not have been due to the decay of the effect, because when retested on Test 1 he reported consistent paradigm CMAEs.

Test 3. Six of the seven remaining Ss reported aftereffects of apparent spatial brightness gradients across the test field, which altered in direction, depending on which way the test field was rotating. Both groups had adapted to fields in which the relatively "brighter above dimmer" field rotated clockwise and the relatively "dimmer above brighter" field rotated counterclockwise. In Test 3 , the test disk was judged to be apparently "dimmer above brighter" when it rotated clockwise and "brighter above dimmer" when it rotated counterclockwise.

Brightness differences could key CMAEs, and transposition effects were found. There was a consistent asymmetry in the results which we cannot explain: With uniformly illuminated fields, a sudden increase in the brightness of the test field produced a CMAE, whereas a sudden decrease did not. With split intensity fields, the asymmetry was less marked, but Ss consistently reported that the brighter of the two halves of the field gave the larger CMAE. The same adaptation to moving fields of different brightness gave two effects: the CMAE produced by test fields of different brightness, and the aftereffect, namely apparent brightness, produced by moving test fields. This was the brightness analogue of the colored aftereffects reported by Hepler and by Stromeyer and Mansfield (1970).

Incidentally, we have also noticed a brightness analogue of McCullough's effect. Inspection of a bright vertical grating alternating with a dim horizontal grating made a vertical test grating look dim and a horizontal test grating look bright.

\section{MAEs CONTINGENT ON SPATIAL FREQUENCY (STRIPE WIDTH) EXPERIMENT 7}

There were $12 \mathrm{Ss}$ in two groups of 6 each.

\section{Apparatus}

The adapting fields consisted of two stationary windows, side by side in a vertical black screen $2.9 \mathrm{~m}$ away from $S$, with a fixation point centered between them. Each window was $25 \mathrm{~cm}$ ( $5 \mathrm{deg}$ of visual angle) high $\mathrm{x}$ $13 \mathrm{~cm}$ (2.5 deg of visual angle) wide, with their inner edges $3.75 \mathrm{~cm}$ (1.3 deg of visual angle) apart. Behind the left window, broad horizontal stripes mounted on a kymograph drum moved upwards at a surface speed of $3 \mathrm{~cm} / \mathrm{sec}(0.6 \mathrm{deg} / \mathrm{sec})$, and behind the right window, narrow horizontal stripes mounted on a second drum moved downwards at the same speed. Every $10 \mathrm{sec}$, the adapting fields were optically reversed from left to right, by means of a Dove prism inserted into the line of sight of each eye. This arrangement exposed each stimulated part of the retina to broad stripes moving up, alternating with narrow stripes moving down.

\section{Adaptation Procedure}

The adapting and test fields were patterns of horizontal black and white stripes of equal mark-space ratios. Group 1 adapted to medium width $1.25-\mathrm{cm}$ ( 2 cycles/deg) stripes moving up, and to narrow $0.625-\mathrm{cm}$ (4 cycles/deg) stripes moving down. Group 2 adapted to broad $2.5-\mathrm{cm}$ (1 cycle/deg) stripes moving up, and to medium $1.25-\mathrm{cm}$ ( 2 cycles/deg) stripes moving down. The luminance of the black stripes was $0.42 \log \mathrm{fL}$ and that of the white stripes was $1.3 \mathrm{log} \mathrm{fL}$.

After $10 \mathrm{~min}$ adaptation, there was a test prccedure (Test 1) followed by a 1-min rest, followed by a further $10 \mathrm{~min}$ adaptation and another test procedure (Test 2).

\section{Test Procedure}

There were two test procedures for which the drums were stopped and the stationary field reversed from left to right approximately every 5 sec. Test 1 was for the paradigm CMAE, and the test field was the stationary adapting field. Test 2 was for the transposition of the CMAE, and the test field was a stationary version of the adapting field of the other $S$ group, alternating left to right every $5 \mathrm{sec}$. Facsimiles of the adapting patterns were pasted onto a piece of cardboard that was positioned behind the windows in the black screen during the test conditions. We ensured that the CMAEs reported were actually caused by the stripe widths and not artifactually induced by the Dove prisms themselves by including control runs on which the stationary test fields were reversed left to right every $5 \mathrm{sec}$ manually by the $E$. The same CMAEs were found as when the prisms were used. During the test procedures, Ss were required to comment on any apparent movement of the fields. They were instructed to name the side of the field and the direction of any apparent motion. If they saw no apparent movement, they were to say so. The adaptation and test stimulus conditions were as follows :

\section{Group 1}

Adaptation. Medium-up and narrow-down, reversed left to right every 10 sec.

Test 1. Stationary medium and narrow, reversed left to right every 5 sec.

Test 2. Stationary broad and medium, reversed left to right every 5 sec.

$$
\text { Group } 2
$$

Adaptation. Broad-up and medium-down, reversed left to right every 10 sec.

Test 1. Stationary broad and medium, reversed left to right every 5 sec.

Test 2. Stationary medium and narrow, reversed left to right every 5 sec.

As can be seen, both groups adapted to the wider stripes moving up and to the narrower stripes moving down.

\section{Results}

The results are shown diagrammatically in Fig. 1.

Test 1. Ten of the $12 \mathrm{Ss}$ reported paradigm CMAEs. They consistently reported (a minimum of 10 successive alternations) an upward MAE on the narrower stripes and a downward MAE on the wider stripes independently of which side of the visual field they were on. Two Ss from Group 2 reported no apparent movement on the test fields, even after the second adaptation period. They did not take Test 2 .

Test 2. Five Ss reported transposed CMAEs. Three Ss from Group 1 and two Ss from the remainder of Group 2 reported no transposed CMAEs. Their reports were inconsistent, but primarily they reported the fields as stationary. When retested on Test 1 , they reported paradigm CMAEs.

It was during this experiment that we discovered quite fortuitously that the size of the CMAE increased after a rest. A previously run $S$, who had waited outside the experimenting room while his friend was being run, was given a retest. He reported the effects as still present and of increased size. Subsequently two out of three other Ss were retested half an hour after completion of adaptation reported the size of the CMAE as increased; one was not certain. In a related study, not reported here, in which we were investigating MAEs contingent on patterns of different sized dots, we found that in general transposition of the CMAE occurred only if a rest period of about $20 \mathrm{~min}$ was given between the adaptation and test procedures. We have no explanation for this phenomenon. 


\section{EXPERIMENT 8}

There were four Ss in one group.

Apparatus

The apparatus was the same as in Experiment 7.

\section{Adaptation Procedure}

The adapting fields were a $1.25 \times$ $2.5 \mathrm{~cm}$ rectangular checkerboard pattern moving up and a $1.25-\mathrm{cm}$ horizontally striped pattern moving down. The fields were reversed left to right every $10 \mathrm{sec}$, so that the stimulated areas of the retina received checkerboard and stripes alternately. The luminances were as in Experiment 7 . There was one adaptation block lasting $10 \mathrm{~min}$ followed by a test procedure.

\section{Test Procedure}

There was one test procedure given on the stationary adapting fields. They were reversed left to right every $5 \mathrm{sec}$ As before, Ss were required to report any apparent movement of the fields.

\section{Results}

All Ss reported consistent CMAEs. The checkerboard elicited a downwards MAE and the striped pattern elicited an upwards MAE.

Stromeyer and Mansfield (1970) suggested that CMAEs may be caused by selective adaptation of "double-duty" units in the visual system that are sensitive to color and movement or color and contour orientation. There is much evidence for the existence of movement-sensitive units (Hubel \& Wiesel, 1959; Barlow \& Hill, 1963) and for orientation-sensitive units or "grating detectors" (Pantle \& Sekuler, 1969, Blakemore \& Campbell, 1969 Blakemore \& Sutton, 1969). It is not difficult to devise models of populations of double-duty units selective to both colors and movement, with some units responding to red-movement-upwards, others to red-down, green-up, green-down, etc. Then selective adaptation of red-up (and green-down) detectors would give a greenish-blue aftereffect on a black and white field moving up, stromeyer found, and an upwards aftereffect on a stationary red test field, as we found.

The demonstration of MAEs contingent on stripes and on checkerboards in Experiment 8 would appear to cast doubts on such an explanation. It seems unlikely that neural units should exist which respond specifically to the direction of movement of checkerboards. And since the checkerboard was cut from the same stripes as the pattern to which it was opposed, it is surprising that the adapting procedure should have induced oppositional CMAEs.

An alternative explanation of these results, and one that we consider more likely, is that the checkerboard effectively consists of a moving horizontal grating plus a stationary superimposed vertical grating. The key for the CMAE would then be the presence of this stationary grating, and an interpretation of the findings of Experiment 8 based on "double-duty" units sensitive to contour orientation and to movement becomes feasible.

This alternative possibility was tested and confirmed, albeit indirectly, in the experiment that follows. Ss adapted to stationary, vertical, and horizontal gratings superimposed on rotary movement, and observed appropriate CMAEs when exposed to stationary vertical or horizontal test gratings.

\section{MAEs OF ROTATION CONTINGENT ON THE ORIENTATION OF STATIONARY GRATINGS EXPERIMENT 9}

There were four $\mathrm{Ss}$ in one group.

\section{Apparatus}

The apparatus was the same as in Experiment 1 for MAEs contingent on color. Briefly, the Ss viewed a patterned disk, $18 \mathrm{~cm}$ in diam, from a distance of $105 \mathrm{~cm}$ through a lightproof tunnel. During the adaptation period, the disk rotated at a speed of $5 \mathrm{rpm}$, alternating in direction every 10 sec. It was back-jlluminated by projectors carrying striped slides and Polaroid filters, as described below.

\section{Adaptation Procedure}

Stationary horizontal or vertical gratings were projected onto the rotating disk. The orientation of the grating was changed each time the disk alternated in direction. All Ss adapted to vertical-clockwise alternating with horizontal-counterclockwise. A possible artifact here was the sheltering of strips of the retina by dark bars in the two gratings, which might set up plaid-like areas on the retina which were unequally adapted to clockwise and counterclockwise movement. If bars in the stationary test fields happened to coincide with these sheltered areas, this might select out a simple MAE from these areas (Harris \& Gibson, 1968). To guard against this possibility, we arranged for the dark bars of each stationary grating to become light and the light bars dark, twice every second. This was done by making the gratings out of strips of Polaroid filter, with alternate strips arranged at orthogonal polarization. A Polaroid filter was rotated in front of the projectors, causing the gratings to reverse their brightness twice per second. The spatial frequency of the gratings was 0.75 cycles/deg. At maximum contrast, the luminances of the dark and light bars were 0.35 and $1,2 \log \mathrm{fL}$, respectively, and the luminance of the spiral not covered by the dark bars was $0.15 \mathrm{log}$ fL.

After $10 \mathrm{~min}$ adaptation, two tests were given. In Test 1 , the stationary gratings projected onto the now-stationary disks cycled in brightness as they had during the adaptation procedure, and in Test 2 the gratings were fixed at their maximum contrast. In both tests, the orientation of the gratings was alternated every 5 sec.

\section{Results}

All Ss consistently reported MAEs whose directions were contingent on the orientation of the gratings. The size of the CMAE was judged to be greater when the grating cycled than when its brightness was fixed. This was perhaps a movement analogue of McCullough's orientation-contingent color aftereffect.

\section{INTEROCULAR TRANSFER OF CMAE \\ EXPERIMENT 10}

There were eight $\mathrm{Ss}$ in two groups of four each.

Apparatus

The apparatus was the same as in Experiments 1 and 9.

Procedure
Group 1 was tested for the interocular transfer of a color-contingent MAE, and Group 2 was tested for the interocular transfer of a MAE contingent on the orientation of stationary superimposed gratings.

The procedure for Group 1 was as follows: Each $\mathrm{S}$ adapted his right eye only to red movement clockwise, alternating every $10 \mathrm{sec}$ with green movement counterclockwise. He was then given a test for the paradigm CMAE, first with his right eye and then with his left eye to test for interocular transfer. He was then retested on his right eye to check that any failure to report interocular transfer was not due to the effect's having decayed. The $S$ then adapted his left eye only, but in the opposite sense, namely, green movement clockwise alternating with red movement counterclockwise. He was then given a test for the CMAE, first with his left eye and then with his right eye, to find out whether opposite and independent CMAEs could now be elicited from each eye.

The procedure (and results) for 
Group 2 were identical, except that movements were paired with vertical and horizontal stationary gratings instead of with red and green colors. Each period of adaptation was for $10 \mathrm{~min}$, and the fields were alternated every $10 \mathrm{sec}$. The testing procedure was as described in earlier experiments.

\section{Results}

In the test for the interocular transfer of the CMAE, all Ss reported no apparent movement on the stationary test fields with the left (unstimulated) eye, but consistently reported CMAEs when viewing the test fields with the right (stimulated) eye. We conclude that there was no interocular transfer of the CMAE. After the second adaptation procedure, all Ss reported an opposite and independent CMAE from each eye. A stationary red test field appeared to rotate counterclockwise when viewed with the right eye and clockwise when viewed with the left eye. A green test field appeared to rotate clockwise when viewed with the right eye and counterclockwise when viewed with the left eye Corresponding results were obtained for Group 2 with the stationary superimposed gratings.

These results suggest that the site of the CMAE is either peripheral to the point of binocular fusion or else pertains to monocularly driven central units.

A point worthy of notice is the durability of the CMAE induced in the right eye. By the time the experiment was completed, this CMAE had lasted some $15 \mathrm{~min}$. Several Ss spontaneously reported it as having increased during the period in which the left eye was being adapted, and as being the larger of the two effects. This is in accord with the findings previously reported in which $S s$ retested $1 / 2 \mathrm{~h}$ after the completion of the adaptation procedures reported an increase in the size of the effects. Of course, these are qualitative subjective reports, but they have occurred with enough regularity to establish the phenomenon.

\section{DISCUSSION}

The CMAEs we have described are new members in a growing class of contingent aftereffects; at least half a dozen of these have been reported (Table 2), and doubtless more await discovery. As yet, we have no explanation for the existence of CMAEs; we can only speculate on the possible lines that a future explanation could take. In our opinion, the most important theoretical question is whether (1) CMAEs are caused by the adaptation of hypothetical "built-in" double-duty units in the visual system, with either fixed or labile response characteristics, or whether (2) the CMAEs are "built up" during the adaptation period by some kind of "perceptual learning" process.

The first hypothesis draws an analogy between CMAEs and simple MAEs. These latter aftereffects can probably be attributed to the adaptation of single-unit movement detectors (Barlow \& Hill, 1963). Other simple AEs of contour orientation, etc., can probably be explained in a similar way. But CMAEs are very different from simple MAEs in their time characteristics:

(1) The CMAE could be very durable, lasting days and even weeks. Stromeyer and Mansfield (1970) reported a motion-contingent colored aftereffect that was still capable of being elicited 6 weeks after a $20-\mathrm{min}$ adaptation procedure. Though the total duration of a CMAE is very large, we found that it appeared only briefly for 1-2 sec after each change of the test fields. Unlike the simple aftereffect, which is always strongest immediately after adaptation and decays away monotonically never to return, the CMAE, though much smaller, could be elicited time and time again.

(2) The CMAE was remarkably indestructible. It seemed immune to degradation by repeated exposure to the adapting stimulus dimensions when they were presented separately. In Experiment 4, after adaptation to red-clockwise alternating with green-counterclockwise, Ss were exposed to stationary red and green fields interposed with white fields which periodically rotated clock- and counterclockwise for a total of $17.5 \mathrm{~min}$. At the end of this procedure, Ss reported a decrease in the size of the CMAE, but it was restored by a 2-min rest in the dark and was reported as being still present, though very small, 2 days later. This is not characteristic of simple aftereffects.

(3) The size of the CMAE increased after a rest. Ss who were retested $1 / 2 h$ after the completion of the normal adaptation and test procedures reported an increase in the size of the effect. And though after repeated exposure to the test fields the CMAE would decrease and even disappear, it would reappear after a short rest. Such behavior bears more resemblance to the phenomenon of "spontaneous recovery" that characterizes the extinction of a conditioned response than to the time course for the recovery from a simple aftereffect.

To summarize, simple MAEs are "one-shot," monotonically decaying processes elicited by a steady-state test field; CMAEs are repetitive, brief, oppositional processes elicited by sudden changes in the brightness, pattern (stripe width), or color of the test fields, which suggests that they may be transient rate-sensitive processes.

If CMAEs and other CAEs are to be attributed to "double-duty" visual units sensitive to a combination of color and movement, etc., there would have to be a separate class of such units for each variety of $\mathrm{CAE}$, each class being sensitive to a pair of inputs (corresponding to each filled-in cell of the matrix in Table 2). Such units, if they existed, could mediate complementary pairs of CAEs-a single adaptation of color and movement units could mediate both color-contingent MAEs and also movement-contingent colored AEs. The existence of these hypothetical visual units in man has been made slightly more plausible by the discovery of multisensory units in the cat cortex, responding to tactile, thermal, and gustatory stimul (Landgren, 1957), visual, auditory, and somatic stimuli (Buser \& Imbert, 1961), and visual, auditory, and labyrinthine stimuli (Jung, Kornhuber, $\&$ da Fonseca, 1963), and of units in the cat superior colliculus sensitive to auditory and visual stimuli (Wickelgren, 1971).

However, these physiological speculations should be treated with caution. They do not account for the transposition effects we found or for the temporal characteristics of CMAEs, which resemble those of conditioning and extinction more than those of physiological adaptation. An alternative explanation for CMAEs and other CAEs is that the association between, say, color and movement is not a function of the fixed inputs to special "built-in" visual units, but is "built up" during the adaptation period by some perceptual learning process, which involves the detection of correlations and contingencies in the visual world. CAEs may thus be an example of the visual system's ability to extract perceptual invariants and redundancies from the visual array (Gibson, 1966). However, there are constraints on the stimuli that give CMAEs. For instance, we obtained CMAEs contingent on spatial frequency (stripe width) but not on shape, such as arrangements of small disks vs small triangles. The only visual dimensions that we have so far found to give CMAEs when paired were those that gave simple AEs when presented singly. So any learning processes underlying CMAEs would have to be different from ordinary forms of conditioning and learning, in which any arbitrarily chosen stimuli can be paired. 


\section{REFERENCES}

ANSTIS, S. M. Visual adeptation to gradual change of intensity. Science, 1967,155 710-712.

BARLOW, H. B., HILL, R. M. Evidence for a physiological explanation of the waterfall phenomenon and figural after-effects. Nature, London, 1963, 200. 1345-1347.

BLAKEMORE, C., \& CAMPBELL, F. W. On the existence of neurons in the human visual system selectively sensitive to the orientation and size of retinal images. Journal of Physiology, London, 1969, $203,237$.

BLAKEMORE, C., \& SUTTON, P. Size adaptation: A new after-effect. Science. $1969,166,245-247$

BUSER, $P_{\text {.. \& IMBERT、 }}$. Sensory projections to the motor cortex in cats: $A$ microelectrode study. In W. A Rosenblith (Ed.), Sensory communication. New York and London: M.I.T. Press and Wiley, 1961 Pp. 597-626.

CAMPBELL, F. W., \& MAFFEI, L. The tilt after-effect: A fresh look. Vision Research, 1971, 11, 833-840.

FAVREAU, 0 EMERSON V \& CORBALLIS, $\dot{M}$. Movement aftereffects contingent on color. Science, 1972, in press.

FIDELL, L. S. Orientation specificity in chromatic adaptation of human "edge-detectors," Perception \& Psy chophysics, 1970, 8, 235-237.

GIBSON, J. J. Adaptation, after-effects, and contrast in the perception of curved lines. Journal of Experimental Psychology. $1933,16,1-31$
GIBSON, J. J. Adaptation with negative after-effect. Psychological Review, 1937, 44. 222-244.

GIBSON, J. J. The senses considered as perceptual systems. Boston: Houghton-Mifflin, 1966.

HARRIS, C. S Effect of viewing distance on a color aftereffect specific to spatial frequency. Paper presented to the Psychonomic Society, San Antonio, Texas, November 1970.

HARRIS, $C$ S \& GIBSON, A R Is orientation-specific color in human vision due to edge-detectors, after-images, or "dipoles"? Science, 1968, 16, 1506-1507.

HELD, R., \& SHATTUCK, S. R. Color- and edge-sensitive channels in the human visual system: Tuning for orientation. Science, 1971, 174, 314-316.

HEPLER, N. Color: A motion contingent after-effect. Science, $1968,162,376-377$.

HOLLAND, H. C. The spiral after-effect. Oxford: Pergamon Press, 1965.

HUBEL, D. H., \& WIESEL, T. N. Receptive fields of single neurones in the cat's striate cortex. Journal of Physiology, $1959,148,574-591$.

HUBEI D $\mathrm{H}$ \& WIESEI, T N. Receptive fields, binocular interaction and functional architecture in the cat's visual cortex. Journal of Physiology, 1962, 160 , 106-154.

JUNG, R., KORNHUBER, H. H., \& da FONSECA, J S. Multisensory convergence on cortical neurons: Neuronal effects of visual, acoustic and vestibular stimuli in the superior convolutions of the cat's cortex. In $G$. Moruzzi (Ed.), Brain mechanisms. Vol. 1. Progress in brain research. Amsterdam,
London, and New York: Elsevier, 1963. Pp. 207-240.

LANDGREN, $S$. Convergence of tactile, thermal and gustatory impulses on single cortical cells. Acta Physiologica Scandinavica, 1957, 40, 210-221.

MCCULLOUGH, C. Color adaptation of edge-detectors in the human visual system. Science, $1965,149,1115-1116$.

PANTLE, A., \& SEKULER, R. W. Contrast response of human visual mechanisms sensitive to orientation and direction of movement. Vision Research, 1969,9, 397-406.

SEKULER, R., \& GANZ, L. After-effect of seen motion with a stabilized retinal image. Science, 1962, 139, 419-420.

STROMEYER, C. F. \& MANSFIELD, R. J. W. Colored aftereffects produced with moving edges. Perception \& Psychophysics, 1970, 7, 108-114.

WICKELGREN, B. Q. Superior colliculus: Some receptive field properties of bimodally responsive cells. Science, 1971 , $173,69-72$.

WOHLGEMUTH, A. On the after-effect of seen movement. British Journal of Psychology Monograph Supplement, $1911,1,1-117$.

\section{NOTE}

1. The test for transposed CMAEs was given on the stationary fields illuminated with the transposed wavelengths.

(Accepted for publication December 31 , 1971.) 